\title{
Effect of pipe size on acetylene flame propagation in a closed straight pipe
}

\section{S.Z. Sulaiman ${ }^{1 *}$, R.M. Kasmani ${ }^{2,3}$, A. Mustafa $^{2}$, S.K. Abdul Mudalip ${ }^{1}$, R. Che Man $^{1}$, S. Md. Shaarani ${ }^{1}$, Z.I. Mohd. Arshad ${ }^{1}$, N.S. Noor Azmi ${ }^{1}$ and N.A.M. Harinder Khan ${ }^{1}$}

\author{
${ }^{1}$ Faculty of Chemical and Natural Resources Engineering, \\ Universiti Malaysia Pahang, 26300 Kuantan, Pahang, Malaysia \\ *Email: szubaidah@ump.edu.my \\ Phone: +6095492357; Fax: +6095492889 \\ ${ }^{2}$ Faculty of Chemical Engineering \& Energy Engineering, \\ Universiti Teknologi Malaysia, 81310 UTM Johor Bahru, Malaysia \\ ${ }^{3}$ Institute of Hydrogen Economy (IHE), Universiti Teknologi Malaysia, \\ 81310 UTM, Johor Bahru, Malaysia
}

\begin{abstract}
The understanding of flame propagation mechanism in a tube or pipe as a function of scale is needed to describe explosion severity. Acetylene is an explosively unstable gas and will lead to a violent explosion when ignited. To achieve the goal, an experimental study of premixed acetylene/air mixture at stoichiometry concentration was carried out in a closed straight pipe with different sizes of L/D (ratio of length to diameter) to examine the flame propagation mechanism. Pipes with $\mathrm{L} / \mathrm{D}=40$ and 51 were used. From the results, it was found that the smaller pipe with $L / D=40$ enhanced the explosion severity by a factor of 1.4 as compared to that of the bigger pipe with $\mathrm{L} / \mathrm{D}=51$. The compression effect at the end of the pipe plays an important role to attenuate the burning rate, leading to higher flame speeds and hence, increases the overpressure. In the case of $\mathrm{L} / \mathrm{D}=40$, the compression effect is more severe due to the larger expansion ratio, and this phenomenon would decrease the quenching effect and subsequently promote flame acceleration. Fast flame speeds of up to $600 \mathrm{~m} / \mathrm{s}$ were measured in the smaller pipe during explosion development. From the results, it can be seen that the compression effect plays a major role in contributing to the higher burning rate and affects the overall explosion and flame speed development. Furthermore, the compression effect is more severe in the smaller pipe that leads to the detonation-like event. This mechanism and data are useful to design a safety device to minimise explosion severity.
\end{abstract}

Keywords: Straight pipe; quenching; compression effect; flame acceleration; detonationlike event.

\section{INTRODUCTION}

It is important to quantify the flame propagation effect and explosion mechanism in order to have an overview of the explosion phenomenon and its effect towards the dynamic flame. Yet, studies have been subjected to numerous researchers over the years [1-8]. These previous works discussed that the presence of an obstacle had a significant effect on the flame propagation in a pipe/channel/tube. The results showed that the presence of an obstacle in the pipe led to randomisation of the flow and thus, enhanced the flame 
speed and overpressure to five times higher than in a straight pipe/tube [9]. Gamezo et al. $[10,11]$ reported that the presence of an obstacle could affect the overall flame propagation mechanism. This is due to the interaction between the reflected shock-wave from the obstacle and the side wall with the flame front that is responsible for the increase of flame surface area, rate of energy release and massive shock strength intensity, and yet turns out to rapid accelerations. In a closed pipe/tube system, the end of pipe wall is considered as an obstacle and both flames or waves will reflect back when reaching the end of pipe due to water hammer effect [12]. When the hot flame and reflective wave interact, they have a tendency to initiate flame perturbation, and affect the explosion behaviour [13]. Experimental results have shown that a closed-end tube is susceptible to induce a flame-reflective wave interaction and enhance the burning rate [14]. Li et al. [15] investigated the flame-reflective wave interaction in a lab-scale explosion test rig using a methane-air mixture at stoichiometric concentration. They stipulated that the interaction between the hot flame and reflective wave plays a major role in contributing to higher peak pressure. Proust [16], in his report on flame acceleration in confined ducts, indicated that hydrogen and ethylene explosion have a different profile towards the end of a pipe event even though both fuels trigger the detonation event. Other than that, Proust [16] also suggested that the interaction between self-acceleration and reflective wave is the main mechanism contributing to the fast flame development. Thomas et al. [17] and Brown et al. [18] observed that flame instabilities in preheated zone result in the flame perturbation leading to flame acceleration and create a secondary explosion. Furthermore, Liberman et al. [19], observed the shock-wave interaction of premixed ethylene/air mixture. They postulated that the compression effect at the end of the wall pipe plays a major role in the flame-reflective acoustic/shock wave interaction contributing to the high burning rate and affects the overall explosion development. Besides flame propagation mechanism, the pipe/vessel size also contributes to flame development. The effect of pipe diameter on flame propagation was studied by Li et al. [20]. They reported that, in smaller pipe diameters, the pressure wave is being induced strongly, resulting in the unburned gases ahead of the flame front to be pre-compressed and pre-heated. This phenomenon causes the laminar burning velocity to increase, and subsequently, flame accelerates rapidly, making the explosion pressure to increase. However, the effect of diameter is insignificant for lean concentration due to the effect of energy loss rate and heat transfer that cause the flame to quench and affect the flame propagation [20]. Furthermore, the effect of tube size towards acetylene/oxygen flame propagation was studied by $\mathrm{Wu}$ et al., [21]. They reported that the tube size has a small influence on flame propagation and reinitiation is the best mechanism to describe flame propagation instead of compression effect or self-acceleration.

The extensive research works mentioned above show that there are still many baffling problems, particularly the mechanism involved in the propagation with respect to pipe size effect. This phenomenon is not well explored and the understanding of this phenomenon should be examined thoroughly, as it has been recognised as one of the factors contributing to the onset of detonation [22, 23].Thus, it is crucial to understand the mechanism causing the flame propagation and leading to detonation hazard in such that, the effective corrective action can be taken. Therefore, this research aims to provide an additional knowledge of flame propagation mechanism (focusing on the pressure and flame speed) in a closed straight pipe of two different sizes using highly reactive fuel. Since acetylene gas is thermodynamically unstable, has a very exothermic behaviour, wide flammability limit (ranging 2.5-81\% v/v) and low ignition energy, it has a potential to initiate detonation hazard $[2,21]$. Therefore, this research aims to provide an additional 
knowledge of flame propagation mechanism (focusing on the pressure and flame speed) in a closed straight pipe of two different sizes using acetylene fuel.

\section{METHODS AND MATERIALS}

\section{Experimental Set-up}

The explosion test was performed in two lab-scale explosion test rigs. The first test rig consisted of a pipe with $5.1 \mathrm{~m}$ long and $0.1 \mathrm{~m}$ diameter (ratio of length to the pipe diameter, L/D was 51) and the second test rig consisted of a pipe with $0.05 \mathrm{~m}$ diameter and $2 \mathrm{~m}$ long giving the L/D as 40. The experimental setup is shown in Figure 1. P1-P6 and T1-T6 are denoted as pressure transducers and thermocouples, used to measure the overpressure and flame speed along the pipe, respectively.

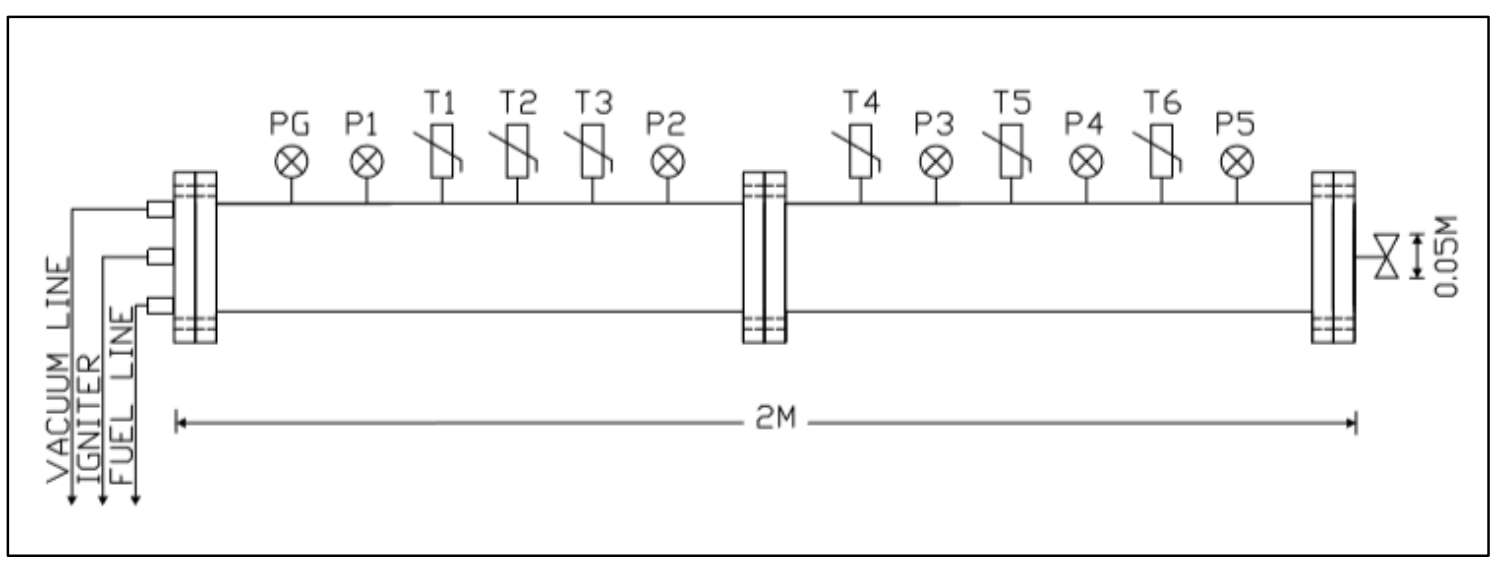

Figure 1. Schematic of a straight pipe.

\section{Gas Mixture}

The tested mixture, acetylene/air was applied at a stoichiometric equivalent ratio $(\phi=1)$, initially at ambient conditions. The gas mixture was mixed directly in the test rig using the partial pressure method. The partial pressure method of mixture preparation added the flammable gas to a vacuum and then added air to approximately 1.013 bar. The explosion was carried out after a delay of about 10 minutes. This method of mixture preparation ensures complete mixing, as the initial vacuum condition rapidly disperses the fuel added and subsequent addition of air takes place under still very low pressure and together with the turbulence from the air injection, rapid mixing is ensured. The mixture composition was controlled to an accuracy of $0.1 \mathrm{mbar}(0.01 \%$ of the composition). The flammable mixture was ignited at the centre of one end of the pipe by means of a spark discharge (ignition energy approximately 16J). The ignition source was placed at the centre of one of the blind flanges.

\section{Sensors and Data Collection}

Pressure measurements were taken at various points along the length of the pipe, using piezoresistive pressure transducers (indicated as P1 to P6 in Figure 1), used to continually measure the pressure development and rates of pressure rise. The pressure transducers were Keller series 11 with an accuracy of $\pm 0.001 \mathrm{~s}$. The history of flame travel along the pipe was recorded by an axial array of mineral insulated, exposed junction, type $\mathrm{K}$ thermocouples CHAL OMEGA (with an accuracy of $\pm 0.001 \mathrm{~s}$ ), along with the centre-line of the entire pipe (indicated as T1-T6 in Figure 1). The flame speed data were generated 
from thermocouple flame arrival times, the time of travel between two adjacent thermocouples and the distance between them. The flame speed data were plotted at the position midway between the thermocouples or in the case of the first flame speed the time between the spark and arrival at the first thermocouple. This technique did not measure the flame temperature as the thermocouple junction was too large $(\sim 0.5 \mathrm{~mm})$, but there was no dead time and the flame was detected as a sudden increase in temperature from a near ambient base temperature. The thermocouple flame arrival time in the pipe was taken to be the first point at which the reading started to rise. With regard to the thermocouples in the pipe, this was hindered by a pre-compression wave ahead of the flame (and associated high flow velocity around the thermocouple) which gave rise to two distinct gradients on the thermocouple trace. In this case, the point at which the second (steeper) gradient became apparent was taken as the flame arrival time. A 32channel with 16-Bit NI CompactDAQ was used to record all the data from the sensor using sampling frequency of $1 \mathrm{kHz}$. Explosion tests (minimum three) were carried out to ensure reproducibility and accuracy.

\section{RESULTS AND DISCUSSION}

\section{Effect of Pipe Size on Acetylene Flame Propagation}

Previous studies showed that pipe sizes have a significant effect on pressure and flame speed $[10,20,24]$. Furthermore, the effect of reactive hydrocarbon fuel such as acetylene has not been fully explored even though acetylene explosions are more violent than other hydrocarbon fuels. Thus, in this section, the mechanism of flame propagation along the pipe was discussed. The discussion focused on pressure development and flame speed as a function of the ratio of the pressure transducer and thermocouple position, (denoted by P1-P5 and T1-T6 in Figure 1) to pipe diameter (X/D).

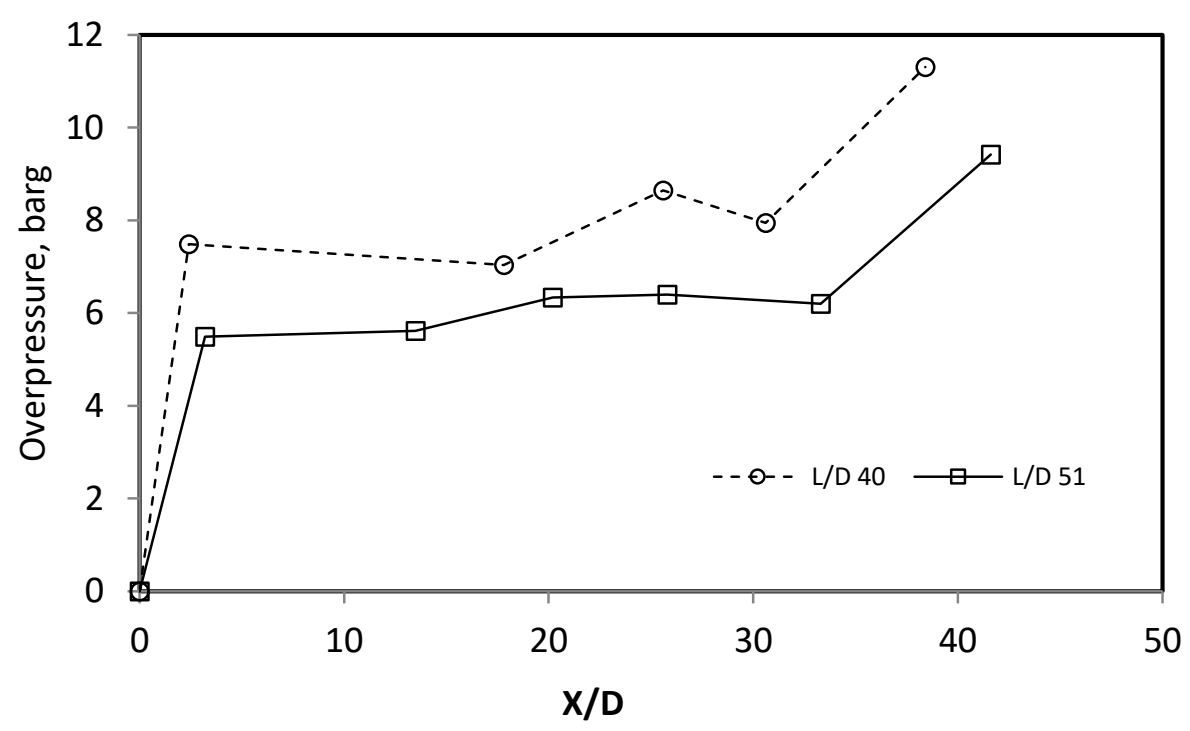

Figure 2. Pressure development along pipe.

\section{Overpressure along Pipe}

As demonstrated in Figure 2, the explosion pressure was more severe in the smaller pipe $(\mathrm{L} / \mathrm{D}=40)$ by a factor of 1.4. Moreover, the time to reach the maximum overpressure for the small pipe ( $\mathrm{L} / \mathrm{D}=40)$ was faster as compared to the bigger pipe $(\mathrm{L} / \mathrm{D}=51)$ (refer to 
Figure 2). According to Li et al., [20], pipe diameter influences explosion mechanism. As the pipe diameter increases, the axial flame propagation also increases. However, the increase of the pipe length decreases the flame speed due to heat losses to the pipe wall [25]. The slower the burning rate, the longer the time for the complete combustion to take place. If combustion occurs over a longer time, it enhances the effect of heat transfer to the pipe wall and causes the flame to be quenched, consequently affecting the pressure development [20]. Furthermore, Xiao et al., [26] stated that the piston effect (compression of the unburned gases) contributes to the flame and pressure development. Thus, it can be said that for pipe with $L / D=40$, the compression effect is more severe due to the larger expansion ratio, and this phenomenon would decrease the quenching effect and subsequently, flame travels at a greater speed, promoting the strong interaction between flame and reflective wave [19].

\section{Flame Speed}

Figure 3 illustrates the flame speed profile along the pipe. Consistent profiles were observed in both pipe sizes, where flame increased gradually before reaching $X / D=25$, but beyond that, a different trend was featured in the result. For the smaller pipe (L/D=40), the flame was decelerated at $\mathrm{X} / \mathrm{D}=25$ before rapid flame propagation began after $\mathrm{X} / \mathrm{D}=30$. This can be explained based on flame extinguishment. According to Iida et al., (1985a and 1985b), the flame front has a tendency to temporarily extinguish in a narrow channel due to stretching and cooling through turbulent mixing before the occurrence of strong burn-up during the reignition. The reignition process causes the flame start to accelerate at a speed of between 115 to $600 \mathrm{~m} / \mathrm{s}$ towards the end of the pipe as shown in Figure 3. However, the reignition process was not performed in the bigger pipe $(\mathrm{L} / \mathrm{D}=51)$ due to the heat loss to the wall that increases with the increase in the L/D or pipe diameter [25] and caused the flame to propagate at a constant velocity towards the end of the pipe. This means that, the flame has a tendency to be quenched rapidly and yet weaken the flame turbulisation. The Re number was approximately $1.37 \times 10^{5}$, which was lower than flame flow in a pipe with $\mathrm{L} / \mathrm{D}=40$, indicating that the flame was more stable and propagated at a constant velocity towards the end of the pipe. It can be concluded that decreasing the pipe size can strengthen the flame propagation as shown on the flame speed profile in Figure 3.

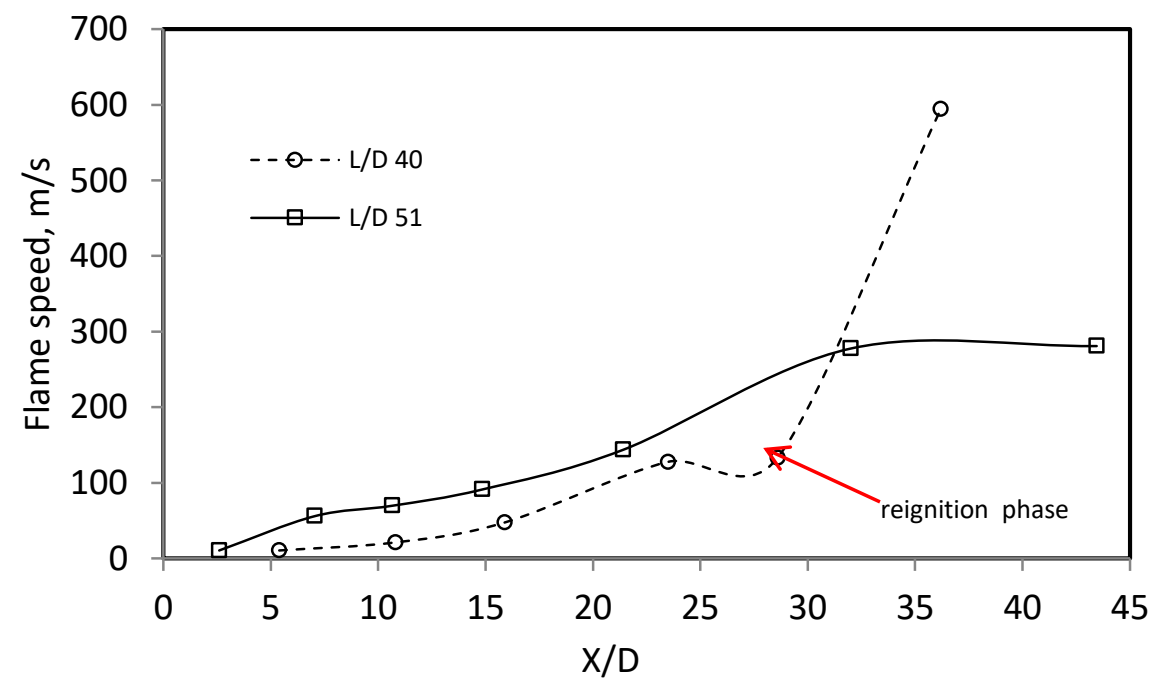

Figure 3. Flame speed along pipe. 


\section{Detonation-like Event in Both Pipe Sizes}

Figure 4 demonstrates the pressure-time histories for both pipe sizes. As shown in the figure, a steep pressure rise was recorded at the end of the pipe. It is very interesting to discuss this phenomenon, since the spiky pressure is not representing the detonation event as the pressure and flame speed are below the Chapman-Jouguet $(\mathrm{CJ})$ condition, in which the CJ pressure is around $15-20$ bar with CJ velocity at $1500-2000 \mathrm{~m} / \mathrm{s}$. The maximum overpressures and velocities obtained from Figure 2 and 3 were 8-11 barg and 300-600 $\mathrm{m} / \mathrm{s}$, respectively. As mentioned earlier, the high compression effect was observed at the end of the pipe (straight pipe), thus, it can be said that the steep pressure rise (detonationlike) was affected by the strong interaction between the hot flame and reflected shock/acoustic wave, due to the highly compressed flame front $[22,27]$. The condition occurs when an initially laminar flame is hit head-on by the shock waves, producing a sudden release of pressure [22]. This release sends a strong rarefaction wave to propagate back into the unburned gases, that creates an unburned gas jet, penetrating the burned gas and developing the shear layers, subsequently produces an extreme turbulence that causes a sudden increase in the burning rate whereby 'trains' of compressed waves are formed $[22,27]$. A study by Karlovitz et al., [28] suggested that the wrinkling of the flame front (turbulisation) can be so great, such that the pockets of trapped unburned gas preheat and collapse in a strong reaction burst. It is possible that such reaction burst may form a single strong shock contributing to a detonation-like wave as illustrated in Figure 4.

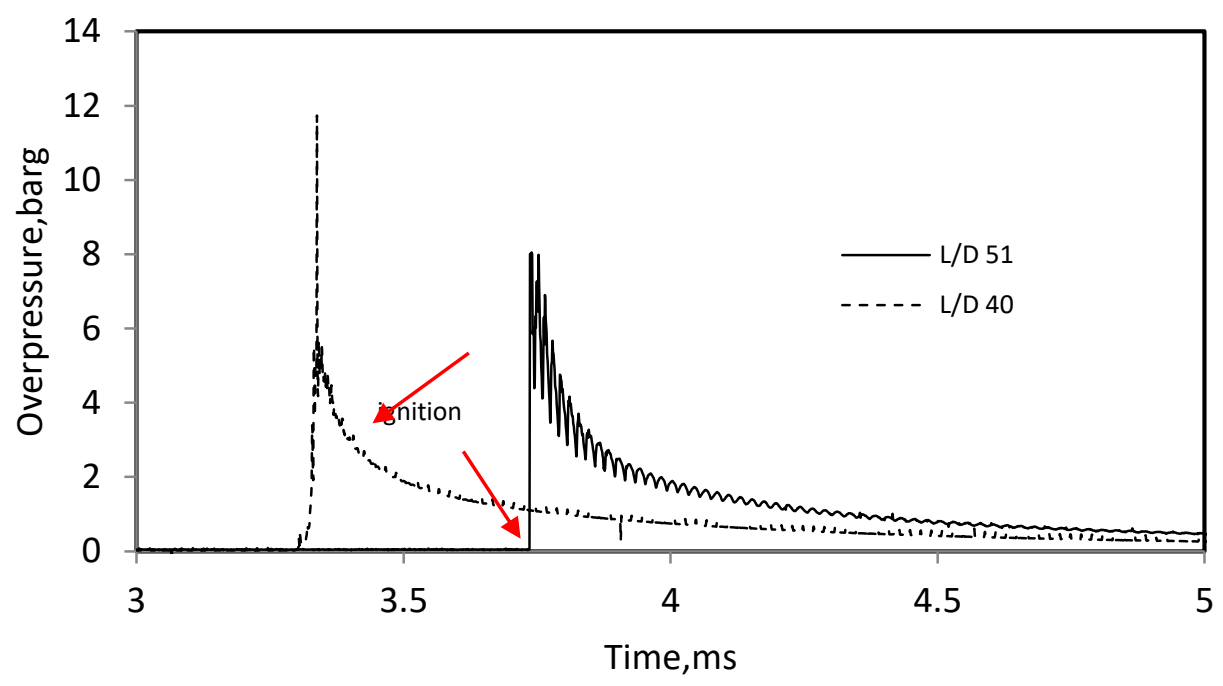

Figure 4. Pressure spike at end of pipe.

In addition, when taking fuel reactivity and dynamic detonation as the basis, premixed acetylene/air explosions have the smallest detonation cell size [29]. The smaller the cell size, the more sensitive is the mixture and it has a quick-chemistry control with rapid exothermic reaction. This condition leads to the increase of $\mathrm{H}$ and $\mathrm{OH}$ radicals in the preheat zone, promotes the reaction and hence, results in the rapid rate of pressure rise. Furthermore, the rapid changes in pressure rise favour the auto ignition and this could further increase the flame propagation, hence, the pressure rises [30]. This means that, the excessive heat release is sufficient enough to maintain the reaction and the adiabatic flame temperature, creating stronger compression waves before the detonation-like event takes place. 


\section{CONCLUSIONS}

In this work, premixed acetylene/air explosions in a closed pipe of different sizes have been studied. The main focus of this research is to study the flame propagation mechanism. The total length of pipe and pipe diameter give a significant effect on the flame propagation development. In a smaller and shorter pipe (diameter $0.05 \mathrm{~m}$, length $2.0 \mathrm{~m}, \mathrm{~L} / \mathrm{D}=40$ ), flame quenching becomes insignificant, leading to the rapid propagation. This situation would strengthen the compression effect and subsequently create a strong turbulent boundary layer between the flame front and the shock/acoustic wave. This phenomenon further amplifies the burning rate and hence, possesses a greater danger to explosion when the hot flame-reflected shock/acoustic wave interact. Since the experiments were conducted in a closed pipe system that was relative to the constantvolume condition, most of the fuel was fully consumed. It is suggested to carry out the open system explosion experiment to best simulate a condition that is experienced in the industrial pipe in case of an explosion.

\section{ACKNOWLEDGEMENTS}

The authors would like to thank the Universiti Teknologi Malaysia for the Research Grant (No; QJ130000 2542 03H41) that allowed development of the explosion test facility. They would also like to thank the Malaysian Ministry of Higher Education (MOHE) for supporting S.Z.Sulaiman's Ph.D. studies.

\section{REFERENCES}

[1] Gwak MC, Yoh JJ. Effect of multi-bend geometry on deflagration to detonation transition of a hydrocarbon-air mixture in tubes. International Journal of Hydrogen Energy. 2013;38:11446-57.

[2] Emami SD, Rajabi M, Che Hassan CR, Hamid MDA, Kasmani RM, Mazangi M. Experimental study on premixed hydrogen/air and hydrogen-methane/air mixtures explosion in 90 degree bend pipeline. International Journal of Hydrogen Energy. 2013;38:14115-20.

[3] Zhu C, Lin B, Jiang B. Flame acceleration of premixed methane/air explosion in parallel pipes. Journal of Loss Prevention in the Process Industries. 2012;25:38390.

[4] Noor MM, Wandel AP, Yusaf T. Effect of Air-Fuel Ratio on Temperature Distribution and Pollutants for Biogas Mild Combustion. International Journal of Automotive and Mechanical Engineering. 2014;10:1980-92.

[5] Noor MM, Wandel AP, Yusaf T. The simulation of biogas combustion in a mild burner. Journal of Mechanical Engineering and Sciences. 2014;6:995-1013.

[6] Feroskhan M, Ismail S. A review on the purification and use of biogas in compression ignition engines. International Journal of Automotive and Mechanical Engineering. 2017;14:4383-400.

[7] Noor M, Wandel AP, Yusaf T. Effect of air-fuel ratio on temperature distribution and pollutants for biogas MILD combustion. International Journal of Automotive and Mechanical Engineering. 2014;10:1980-92.

[8] Noor M, Wandel AP, Yusaf T. The simulation of biogas combustion in a mild burner. Journal of Mechanical Engineering and Sciences. 2014;6:995-1013. 
[9] Phylaktou H, Foley M, Andrews GE. Explosion enhancement through a $90^{\circ}$ curved bend. Journal of Loss Prevention in the Process Industries. 1993;6:21-9.

[10] Gamezo VN, Ogawa T, Oran ES. Numerical simulations of flame propagation and DDT in obstructed channels filled with hydrogen-air mixture. Proceedings of the Combustion Institute. 2007;31:2463-71.

[11] Gamezo VN, Ogawa T, Oran ES. Flame acceleration and DDT in channels with obstacles: Effect of obstacle spacing. Combustion and Flame. 2008;155:302-15.

[12] Oppenheim AK. Dynamic Features of Combustion. Philosophical Transactions of the Royal Society of London Series A, Mathematical and Physical Sciences. 1985;315:471-508.

[13] Xiao H, An W, Duan Q, Sun J. Dynamics of premixed hydrogen/air flame in a closed combustion vessel. International Journal of Hydrogen Energy. 2013;38:12856-64.

[14] Teerling OJ, McIntosh AC, Brindley J, Tam VHY. Premixed flame response to oscillatory pressure waves. Proceedings of the Combustion Institute. 2005;30:1733-40.

[15] Li Q, Lin B, Jian C. Investigation on the Interactions of Gas Explosion Flame and Reflected Pressure Waves in Closed Pipes. Combustion Science and Technology. 2012;184:2154-62.

[16] Proust C. Gas flame acceleration in long ducts. Journal of Loss Prevention in the Process Industries. 2015;36:387-93.

[17] Thomas GO. Flame acceleration and the development of detonation in fueloxygen mixtures at elevated temperatures and pressures. Journal of Hazardous Materials. 2009;163:783-94.

[18] Brown CJ, Thomas GO. Experimental studies of shock-induced ignition and transition to detonation in ethylene and propane mixtures. Combustion and Flame. 1999;117:861-70.

[19] Liberman MA, Ivanov MF, Kiverin AD, Kuznetsov MS, Chukalovsky AA, Rakhimova TV. Deflagration-to-detonation transition in highly reactive combustible mixtures. Acta Astronautica. 2010;67:688-701.

[20] Li J, Lai W, Chung K. Tube diameter effect on deflagration-to-detonation transition of propane-oxygen mixtures. Shock Waves. 2006;16:109-17.

[21] Wu Y, Zheng Q, Weng C. An experimental study on the detonation transmission behaviours in acetylene-oxygen-argon mixtures. Energy. 2018;143:554-61.

[22] Thomas G, Williams RL. Detonation interaction with wedges and bends. Shock Waves. 2002;11:481-92.

[23] Wang C, Han W, Ning J, Yang Y. High resolution numerical simulation of methane explosion in bend ducts. Safety Science. 2012;50:709-17.

[24] Blanchard R, Arndt D, Grätz R, Poli M, Scheider S. Explosions in closed pipes containing baffles and 90 degree bends. Journal of Loss Prevention in the Process Industries. 2010;23:253-9.

[25] Chatrathi K, Going JE, Grandestaff B. Flame propagation in industrial scale piping. Process Safety Progress. 2001;20:286-94.

[26] Xiao H, He X, Duan Q, Luo X, Sun J. An investigation of premixed flame propagation in a closed combustion duct with a $90^{\circ}$ bend. Applied Energy. 2014;134:248-56.

[27] Uchida M, Suda T, Fujimori T, Fujii T, Inagaki T. Pressure loading of detonation waves through 90-degree bend in high pressure H2-O2-N2 mixtures. Proceedings of the Combustion Institute. 2011;33:2327-33. 
[28] Karlovitz B, Denniston DW, Wells FE. Investigation in turbulent flames. Journal of Chemical Physics. 1951;19:541-7.

[29] Lee JHS. Dynamic Parameters of Gaseous Detonations. Annual Review of Fluid Mechanics. 1984;16:311-36.

[30] Egerton A, Gates SF. Further Experiments on Explosions in Gaseous Mixtures of Acetylene, of Hydrogen and of Pentane. Proceedings of the Royal Society of London Series A, Containing Papers of a Mathematical and Physical Character. 1927;116:516-29. 